\title{
Natural history of Ross River virus-induced epidemic polyarthritis
}

\author{
Andrea D Mylonas, Allison M Brown, Tracy L Carthew, Barry McGrath, David M Purdie, Nirmala Pandeya, Philip C Vecchio, \\ Louisa G Collins, lan D Gardner, Ferdinandus J de Looze, Elizabeth J Reymond and Andreas Suhrbier
}

\section{ABSTRACT}

Objective: To describe the natural history, treatment and cost of Ross River virusinduced epidemic polyarthritis (RRV disease).

Design: Questionnaire-based longitudinal prospective study.

Participants and setting: Patients in the greater Brisbane area, Queensland, diagnosed with RRV disease by their general practitioners based on clinical symptoms and paired serological tests between November 1997 and April 1999.

Main outcome measures: Scores on two validated quality-of-life questionnaires (Clinical Health Assessment Questionnaire and Medical Outcomes Study Short Form 36) were obtained soon after diagnosis and one, two, three, six and 12 months thereafter. Scores were compared between patients diagnosed with RRV disease alone and those with RRV disease plus other conditions.

Results: 67 patients were enrolled. Most patients with RRV disease alone had severe acute symptoms, but followed a consistent path to recovery within three to six months. Other conditions, often chronic rheumatic diseases or depression, were identified in half the cohort; their quality-of-life scores suggested stable chronic illness between six and 12 months after diagnosis. Non-steroidal anti-inflammatory drugs (NSAIDs) were taken by $58 \%$ of patients (average use, 7.6 weeks; range, 2-22 weeks). Time off work averaged 1.9 days, and direct cost to the community was estimated as \$A1018 per patient.

Conclusions: Symptom duration and frequency of long-term symptoms may have been overestimated by previous studies of RRV disease. Disease persisting six to 12 months after RRV diagnosis was largely attributable to other conditions, highlighting the need to seek other diagnoses in RRV patients with persistent symptoms.

\section{See also page 352}

Australian Centre for International Health and Nutrition, Queensland Institute of Medical Research, Brisbane, QLD.

Andrea D Mylonas, BA, RN, Research Assistant;

Allison M Brown, RN, Grad Cert CDM, Research Assistant;

Tracy L Carthew, RN, BN, Research Assistant;

David M Purdie, MMedSc, PhD, Biostatistician;

Nirmala Pandeya, GradDipAppSc, MMedSc, Research Assistant;

Louisa G Collins, BEc, MPH, Research Assistant;

Andreas Suhrbier, MA, PhD, Associate Professor.

Queensland Research and Health Promotion Unit, Royal Australian College of General

Practitioners, Brisbane, QLD.

Barry McGrath, MB BS, PhD, Former Director;

Elizabeth J Reymond, FRACGP, PhD, Director.

Department of Rheumatology, Princess Alexandra Hospital, Brisbane, QLD.

Philip C Vecchio, FRACP, MBA, Director.

Department of Immunology, Queensland Medical Laboratory, Brisbane, QLD. Ian D Gardner, PhD, FACTM, Chief Scientist.

University General Practice, University of Queensland, Brisbane, QLD.

Ferdinandus $\mathbf{J}$ de Looze, MB BS, MSc, Senior Lecturer.

Reprints will not be available from the authors. Correspondence: Associate Professor Andreas Suhrbier, Australian Centre for International Health and Nutrition, Queensland Institute of Medical Research,

Post Office, Royal Brisbane Hospital, Brisbane, QLD 4029.

andreasS@ qimr.edu.au
Ross RIVER VIRUS (RRV) is endemic in Australia and New Guinea and is the aetiological agent of epidemic polyarthritis, or RRV disease. In Australia, where this disease is notifiable, up to 8000 cases are reported annually. The principal symptoms are polyarthritis and arthralgia, with about half the patients also experiencing a rash, fever, myalgia or fatigue. ${ }^{1,2}$

Several surveys of RRV disease have produced conflicting data, particularly on duration of disease. ${ }^{1,3-6}$ However, most of these surveys were retrospective, none used objective measures of illhealth, and few sought to differentiate between RRV disease and other potentially confounding diseases. The only determination of the financial burden of RRV disease estimated an epidemic of 1196 cases to cost about $\$$ A3 million. ${ }^{6}$

Our study involved a survey of patients with RRV disease using two validated quality-of-life questionnaires to describe duration and severity of RRV disease more objectively than heretofore. Additional illnesses were documented in consultation with patients and treating doctors. Drug treatments were also recorded, and the direct cost of RRV disease was estimated.

\section{METHODS}

The study was a prospective longitudinal survey carried out in Brisbane between November 1997 and April 2000.

\section{Study population}

Patients were eligible if they were diagnosed with RRV disease by a general practitioner in the greater Brisbane area based on clinical symptoms and results of paired RRV serological tests using standard enzyme-linked immunosorbent assay. They were recruited through requests to GPs printed on all pathology reports from the Queensland Medical Laboratory showing a positive result for 


\section{1: Demographic characteristics of a cohort with Ross River virus disease}

$\begin{array}{lc}\text { Sex distribution (\% female) } & 57 \% \\ \text { Mean age in years (range) } & 41.6(20-89) \\ \begin{array}{l}\text { Age and sex distribution } \\ \text { (male, female) }\end{array} & \\ 20-29 \text { years } & 7 \%, 6 \% \\ 30-39 \text { years } & 12 \%, 22 \% \\ 40-49 \text { years } & 12 \%, 15 \% \\ 50-59 \text { years } & 9 \%, 12 \% \\ 60-69 \text { years } & 2 \%, 2 \% \\ 70-89 \text { years } & 2 \%, 0 \\ \text { Marital status } & \\ \text { Single } & 16 \% \\ \text { Married } & 69 \% \\ \text { Defacto } & 6 \% \\ \text { Divorced } & 9 \% \\ \text { Employment } & \\ \text { Full time } & 66 \% \\ \text { Part time } & 18 \% \\ \text { Unemployed/retired } & \\ \end{array}$

RRV IgM in a patient in the recruitment area. GPs were asked to invite the patients to contact the enrolling nurse. Some patients were recruited through letters sent to GPs listed on the database of the Royal Australian College of General Practitioners.

The study was approved by the ethics committees of the Royal Australian College of General Practitioners, the Queensland Institute of Medical Research, and the Princess Alexandra Hospital, Brisbane.

\section{Patient assessment}

Patients attended an initial interview with a research nurse soon after the first positive IgM result, and were given an information leaflet and asked to sign a consent form. They were followed up one, two, three, six and 12 months later.

At each visit, they were asked to describe their medications, which were confirmed with the treating doctors. They were also asked to complete the Medical Outcomes Study Short Form $36(\mathrm{SF}-36)^{7}$ and the Clinical Health Assessment Questionnaire (CLINHAQ). ${ }^{8}$ The SF-36 questionnaire is used to assess health-related quality of life in a variety of diseases and conditions, ${ }^{9}$ while the CLINHAQ was developed primarily to assess patients with chronic rheumatic disorders. ${ }^{10}$

Premorbid diseases were documented at the first visit, and concurrent diseases at the first and subsequent visits, in consultation with patients and their doctors. Nearly all patients were also reviewed by a rheumatologist (P C V) to identify or confirm concurrent conditions at one to two months after diagnosis and, for those with suspected or confirmed comorbidities, again at three to six months after diagnosis.

\section{Statistical and cost analyses}

At each visit, patients were classified as having a diagnosis of RRV disease alone or RRV disease plus other conditions. Mean quality-of-life scores were compared between the two groups using $t$ tests, the Wilcoxon rank-sum test and ANOVA.

We estimated the direct cost of RRV disease per patient by summing the cost of RRV serological tests (including costs of negative tests), a conservative estimate of other co-ordered tests, lost income from time off work, and costs of medications and GP visits, including patient travel costs.

\section{RESULTS}

\section{Study population}

Sixty-seven patients with RRV disease were enrolled. A further three patients who otherwise met the criteria were excluded as paired serological tests failed to confirm RRV disease. Seven patients left the study, and at each visit up to seven failed to answer all questions.

From data on notified cases by suburb and month (Queensland Health, personal communication), we estimate that the 67 enrolled patients represented about $20 \%$ of patients reported with RRV disease within the recruitment area. We also estimate that about half the GPs in this area received requests to ask their patients to enrol, suggesting that the study captured up to $40 \%$ of available patients.

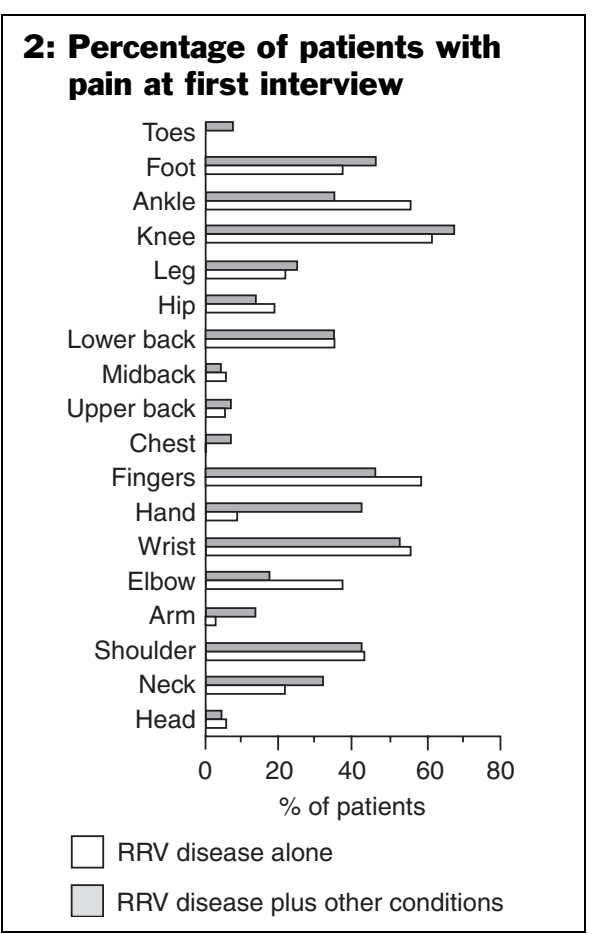

The demographic profile of the cohort (Box 1) was similar to that reported previously for patients with RRV disease. ${ }^{1,3-6}$ The breakdown of occupations did not appear different from that seen in urban populations in Australia. ${ }^{11}$ Patient homes were distributed evenly around the greater Brisbane area.

\section{Comorbidity}

Although some comorbid conditions were identified during the first three months after diagnosis, the full spectrum emerged only at six months after review by the rheumatologist.

At six months after diagnosis, 32 of 60 patients $(53 \%)$ were classified with RRV disease alone. Twenty eight patients had additional diagnoses, including eight with other chronic rheumatic conditions (osteoarthritis, 3; rheumatoid arthritis, 2 ; psoriatic arthritis, 1; ankylosing spondylitis, 1; and osteoarthritis plus psoriatic arthritis, 1). Eight were taking medications for depression, and all but one of these had a history of depression or serious illness before RRV diagnosis. The prevalence of other rheumatic conditions and depression in the RRV disease cohort did not differ significantly from the expected prevalence. ${ }^{12}$ Other diagnoses 


\section{3: Mean scores ( \pm standard error) on two quality-of-life questionnaires for a cohort* with Ross River virus disease Medical Outcomes Study Short Form $36\left(\right.$ SF-36) ${ }^{7 \dagger}$}

RRV disease alone $\quad \square$ RRV disease plus other conditions _ - - - "Norm" (score for age- and sex-matched general Australian population)
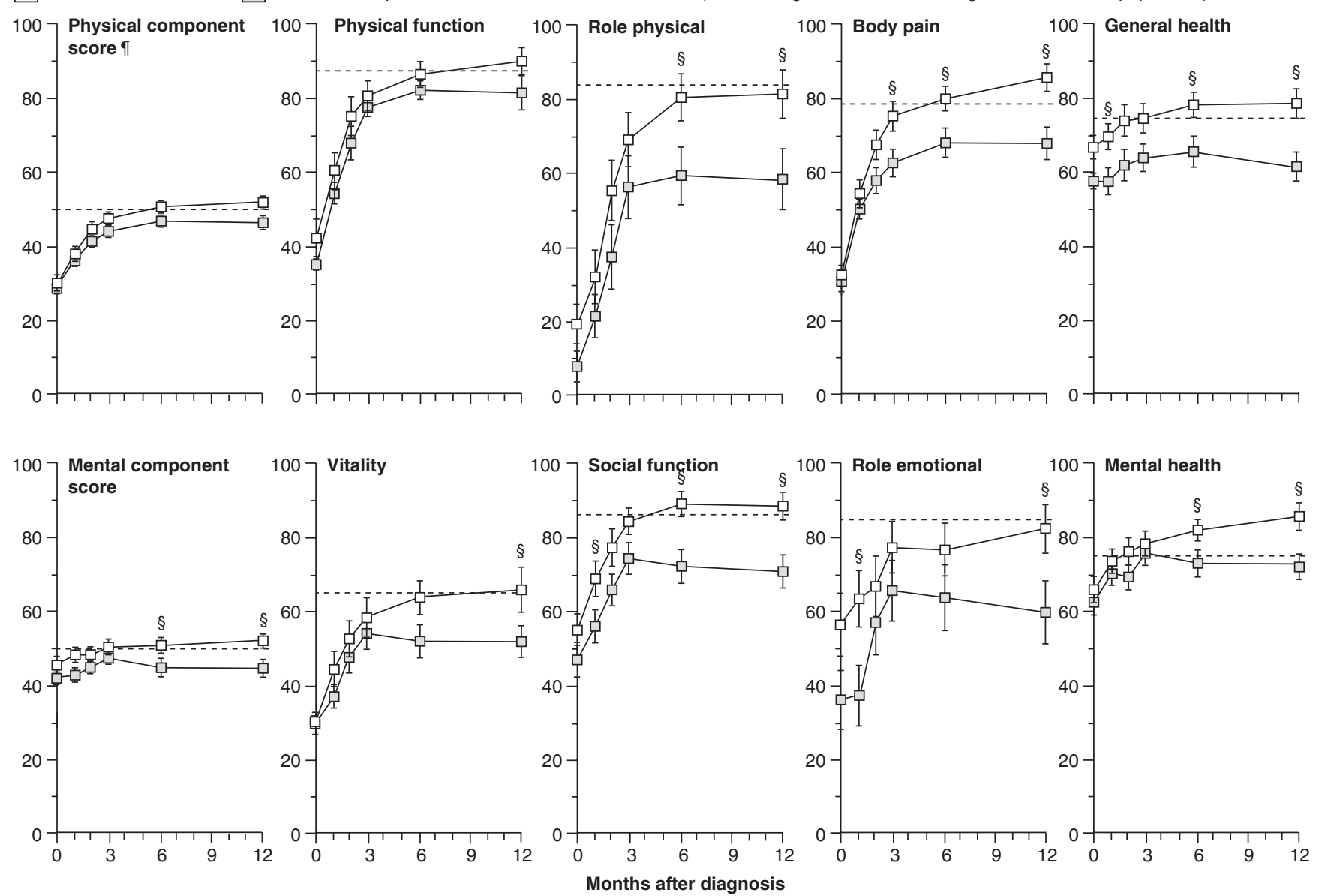

*Survey included 67 patients (0 months), 64 (1 month), 62 (2 months), 61 (3 months), and 60 (6 and 12 months). All questions were completed on both questionnaires by 32 (RRV disease alone) and 28 (RRV disease plus other conditions) (0 months), 31 and 27 ( 1 month), 29 and 25 ( 2 months), 31 and 27 ( 3 months), 29 and 28 (6 months), and 30 and 27 (12 months).

†SF-36 measures health on eight 100-point scales, where higher scores represent poorer health. Physical and Mental component scores are summary scores for the four physical and four emotional domains, respectively. "Role physical" and "Role emotional" represent role limitations due to physical and emotional problems, respectively.

at six months included carpal tunnel syndrome, back pain and obesity, herniated disc, melanoma, pneumonia, hypercholesterolaemia, polycystic ovaries, endometriosis, urinary tract infection, thrombocytopenia, allergy, and pregnancy (all with a prevalence of 1 ).

At 12 months after diagnosis, 29 of 60 patients $(48 \%)$ had additional diagnoses.

\section{Natural history of RRV disease}

Frequency of pain in different joints at first interview is shown in Box 2. Knees, wrists and fingers were most often involved. The percentage of patients whose pain was symmetrical ranged from $36 \%$ (shoulder pain) to $100 \%$ (pain in the hands).

Mean SF-36 and CLINHAQ scores over the 12 months of follow-up are shown in Box 3. There was generally excellent concordance between the two measures, and both instruments illustrated that patients diagnosed with RRV disease alone tended to have a consistent path to recovery within three to six months. In contrast, the scores of patients diagnosed with RRV disease plus another condition usually failed to reach the population norm (defined as the score for the age- and sex-matched general Australian population). These patients usually had significantly worse illness at six and 12 months than those with RRV disease alone and also usually had stable chronic ill-health between the three- and 12-month reviews.

Twelve months after RRV diagnosis, 16 patients still had an SF-36 physical component (PC) score at least 5 points below the norm score of 50 . They comprised:

- 11 patients with conditions in addition to RRV disease, primarily chronic rheumatic conditions or depression; - three patients classified with RRV disease alone who had had significant illnesses before RRV diagnosis - a broken hip, stomach stapling procedure and burns plus pneumonia, respectively 


\section{Clinical Health Assessment Questionnaire (CLINHAQ ${ }^{8 \ddagger}$}

$\square$ RRV disease alone $\quad \square$ RRV disease plus other conditions _ - - - "Norm" (score for age- and sex-matched general Australian population)
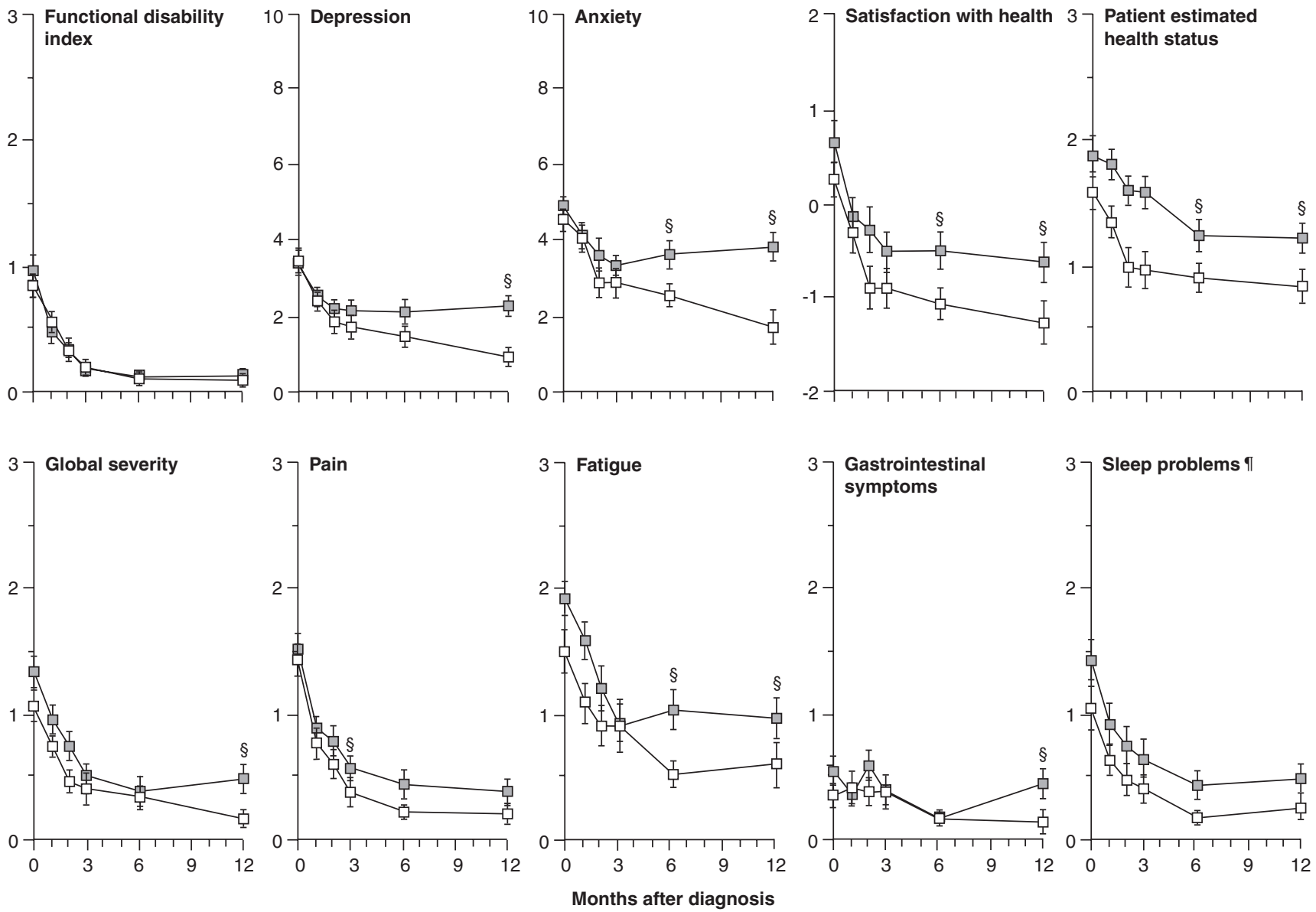

fHigher scores on the CLINHAQ represent poorer health.

$\S$ Scores differed significantly $(P<0.05)$ between patients with RRV disease alone and those with additional conditions, as determined by $t$ test (SF-36 scores) or Wilcoxon rank-sum test (CLINHAQ scores).

ๆPhysical component scores and sleep problem scores differed significantly $(P=0.01)$ between patients with RRV disease alone and those with RRV disease plus other conditions when the overall mean differences for all time points were compared by ANOVA.

(all with PC scores 40-45, indicating marginal disease severity);

one patient who was hospitalised for pneumonia six months after RRV diagnosis (PC score 28); and

- one patient with no other identified conditions (PC score 30), suggesting that RRV disease was the sole cause of symptoms.

\section{Treatment}

Non-steroidal anti-inflammatory drugs (NSAIDs) were taken by $58 \%$ of patients, aspirin or paracetamol by $15 \%$, prescribed corticosteroids by $4 \%$, and NSAIDs plus aspirin or paraceta- mol by $3 \% ; 21 \%$ took no conventional medication. The percentage of patients taking NSAIDs decreased over the 12 months after diagnosis, from 55\% (initial interview), to $31 \%$ ( 1 month), $21 \%$ (2 months), 15\% (3 months), 5\% (6 months), and $2 \%$ (12 months). NSAIDs were taken for an average of 7.6 weeks (SD, 5.9; range, 2-22 weeks). At each visit, from $70 \%$ to $100 \%$ of patients taking NSAIDs reported being satisfied with this treatment.

\section{Direct cost of RRV disease}

The direct cost to the community was estimated to be $\$ A 1018$ per patient, with diagnostic costs and lost productivity representing the major components (Box 4). Average time off work was 1.9 days for the whole cohort (SD, 5.0), 3.1 days for the 43 patients in full-time employment (SD, 6.7; range, 0-37), and 2.4 days for the 35 in full-time employment who had RRV disease alone (SD, 4.3). Twenty-five of those in full-time employment $(58 \%)$ took no time off work.

Costs were not included for mosquito control programs (an estimated $\$ 3$ million for the Brisbane area), indirect costs of RRV disease morbidity, monitoring of RRV as a notifiable disease and RRV research expenditure (about $\$ 200000-\$ 400000$ per annum). 


\section{4: Estimated direct costs of Ross River virus disease per patient}

$\begin{array}{lc}\text { Diagnostic tests* }^{*} & \$ 567(56 \%) \\ \text { Lost productivity }^{\dagger} & \$ 305(30 \%) \\ \text { GP visits }^{\ddagger} & \$ 88(9 \%) \\ \text { Medications }^{\S} & \$ 46(4 \%) \\ \text { Patient travel }^{\uparrow} & \$ 12(1 \%) \\ \text { Total } & \$ 1018\end{array}$

$\mathrm{GP}=$ general practitioner

${ }^{*}$ At $\$ 23.80$ per serological test for Ross River virus (RRV). Estimate includes costs of negative tests (23 patients were tested to detect one case of RRV disease) plus conservative estimate of other coordered tests, based on unpublished data gathered by Queensland Medical Laboratory on 54790 patients between Oct 1997 and Apr 1999. †Based on average gross weekly earnings of $\$ 795.20$ and mean of 1.92 days off work for the whole cohort.

¥Mean of 3.25 visits (SD, 1.12), based on 20 randomly selected patients, at $\$ 27.00$ per visit.

$\S$ Based on 12 representative patients using Pharmaceutical Benefits Scheme costs.

१ Estimated mean of $8 \mathrm{~km}$ per return trip to GP $\times$ cost of medium car $(\$ 0.45 / \mathrm{km}) \times 3.25$ visits

\section{DISCUSSION}

This study illustrates that RRV disease is severe at onset but usually resolves within three to six months. Importantly, patients with continuing chronic disease beyond the three- to six-month followups usually had additional conditions. These observations illustrate the importance of seeking differential diagnoses in patients presenting with long-term RRV disease.

These findings are consistent with those of a 1997 study of 103 Queensland residents with RRV disease, which also noted a high incidence of other rheumatic conditions. ${ }^{14}$ The high incidence of long-term RRV disease and long duration reported by other studies ${ }^{5}$ may be somewhat overestimated and unduly influenced by other chronic conditions. In our study, RRV disease lasting over a year was identified in only one patient with no confounding conditions ( $2 \%$ of the cohort).

At diagnosis, patients with RRV disease had SF-36 scores comparable to those of 55-64-year-olds with osteoarthritis awaiting hip or knee replacement surgery. ${ }^{15}$ Scores for both groups were 50-70 points lower than the norm for the domains role physical, physical function and body pain; 25-35 points lower for vitality, social function and role emotional; and 10-20 points lower for general and mental health. At diagnosis, our cohort also had similar SF-36 physical and mental component scores as patients with persistent Lyme disease, ${ }^{16}$ and similar average functional disability index and fatigue and pain scores as a US cohort of patients with chronic rheumatoid arthritis. ${ }^{7,17}$ Depression and anxiety scores in our patients at diagnosis approached the cutoff scores for clinical depression and anxiety (about 4 and 6, respectively) and were similar to those found in patients with rheumatoid arthritis three months after onset. ${ }^{17}$ Sleep problems have not been widely associated with RRV disease, but were also similar to those seen in chronic rheumatoid arthritis. $^{17}$

A potential limitation of our study was the inability to enrol a greater percentage of eligible patients with RRV disease. The study was also of insufficient size to establish whether the single case of long-term RRV disease represents the true frequency.

To our knowledge, this study represents the first published use of validated questionnaires for investigating a viral arthritis and demonstrates their value for assessing these diseases. The results clearly highlight the need to consider alternative diagnoses in patients with persisting RRV disease symptoms. Patients with RRV disease can also be reassured that the disease, although severe at onset, is self-limiting, with a usual duration of three to six months, and does not have long-term sequelae.

\section{ACKNOWLEDGEMENTS}

We would like to thank the pathology companies Queensland Medical Laboratory and Sullivan and Nicolaides, Brisbane, QLD), Dr F Wolfe (Director, National Data Bank for Rheumatic Diseases, Kansas, USA), Dr J F Farmer (Brisbane), M Harris and Professor B Kay (Queensland Institute of Medical Research, Brisbane) for their contributions. The research was funded by Queensland Health, the Australian Rotary Health Research Fund (Arbovirus Prevention Research Grants), the Australian National Centre for International and Tropical Health and Nutrition, and the Queensland Institute of Medical Research Trust.

\section{COMPETING INTERESTS}

None declared.

\section{REFERENCES}

1. Fraser JR. Epidemic polyarthritis and Ross River virus disease. Clin Rheum Dis 1986; 12: 369-388.

2. Flexman JP, Smith DW, Mackenzie JS, et al. A comparison of the diseases caused by Ross River virus and Barmah Forest virus. Med J Aust 1998; 169: 159-163.

3. Westley-Wise VJ, Beard JR, Sladden TJ, et al. Ross River virus infection on the North Coast of New South Wales. Aust N Z J Public Health 1996; 20: 87-92.

4. Selden SM, Cameron AS. Changing epidemiology of Ross River virus disease in South Australia. Med J Aust 1996; 165: 313-317.

5. Condon RJ, Rous IL. Acute symptoms and sequelae of Ross River virus infection in South-Western Australia: A follow-up study. Clin Diag Virol 1995; 3: 273-284.

6. Hawkes RA, Boughton CR, Naim HM, Stallman ND. A major outbreak of epidemic polyarthritis in New South Wales during the summer of $1983 / 1984$. Med J Aust 1985; 143: 330-333.

7. Medical Outcomes Study Short Form 36 (SF-36). Available at http://www.hsru.ox.ac.uk/shortfrm.htm Accessed 1997

8. Arthritis Research Center Foundation. Clinical Health Assessment Questionnaire (CLINHAQ). Available at http://www.arthritis-research.org/clinhaq.htm Accessed 1997.

9. Australian Bureau of Statistics. National health survey Australia, 1995: SF-36 population norms. Canberra, ABS, 1997. (Catalogue No. 4399.0.)

10. Wolfe F, O'Dell JR, Kavanaugh A, et al. Evaluating severity and status in rheumatoid arthritis. J Rheumatol 2001; 28: 1453-1462.

11. McLennan W. Australian social trends 2001. Canberra: ABS. (Catalogue No. 4102.0.)

12. Murtagh JE. General practice. 2nd ed. Sydney: McGraw-Hill. 1998.

13. Australian Bureau of Statistics. Average weekly Australian earnings. Canberra: ABS, 2000. (Catalogue No. 6302.)

14. Keary JP. Review of 103 patients with RRV EPA. In: Abstracts of the Australian Rheumatology Association 40th Annual Scientific Conference. May 1997; Brisbane, QLD. Sydney: ARA, 1997.

15. March LM, Cross MJ, Lapsley H. Outcomes after hip or knee replacement surgery for osteoarthritis. A prospective cohort study comparing patients' quality of life before and after surgery with age-related population norms. Med J Aust 1999; 171: 235-238.

16. Klempner MS, Hu LT, Evans J, et al. Two controlled trials of antibiotic treatment in patients with persistent symptoms and a history of Lyme disease. N Engl J Med 2001; 345: 85-92.

17. Wolfe F, Skevington SM. Measuring the epidemiology of distress: the rheumatology distress index. $J$ Rheumatol 2000; 27: 2000-2009. 